\title{
Estudio de la epidemia de gripe A nH1N1 de 2009-2010. Síntomas guía en Atención Primaria. Comparación clínica con los casos hospitalarios
}

\author{
N. Martín Ruiza ,J. Galbe Sánchez-Venturab , N. García Sánchezc, MÁ. Ruiz Andrés ${ }^{d}$ \\ ${ }^{a}$ MIR-Pediatría. Servicio de Pediatría. Hospital Miguel Servet. Zaragoza. España.

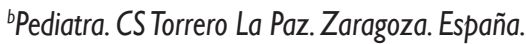 \\ 'Pediatra. CS Delicias Sur. Zaragoza. España. \\ ¿Servicio de Microbiología. Hospital Miguel Servet. Zaragoza. España.
}

\section{Resumen}

Objetivos: analizar la capacidad de predicción clínica respecto al diagnóstico de gripe A nH1N1 de los sintomas que presentan los pacientes ambulatorios registrados en la base de datos de la Red Centinela de Aragón (RCA). Comparar la casuística ambulatoria con los pacientes ingresados durante la epidemia de gripe A nH1N1 (2009-2010).

Pacientes y métodos: estudio descriptivo retrospectivo de los pacientes que cumplían los criterios epidemiológicos de definición de caso, en los que de manera aleatoria se les realizó reacción en cadena de la polimerasa en tiempo real (PCR-RT) de gripe A nH1N1, así como de los pacientes ingresados en el hospital de tercer nivel de referencia. Comparación de las características epidemiológicas y clínicas entre los pacientes ambulatorios y hospitalarios.

Resultados: de los pacientes registrados por la RCA no se encontraron diferencias clínicas entre los niños con frotis (PCR-RT) positivo o negativo para el virus gripal nH1N1, por lo que no se ha podido encontrar un conjunto de síntomas que sean predictores de tener un frotis positivo con la $P C R-R T$. Los pacientes hospitalizados tenían menor edad que los de la RCA, así como mayor porcentaje de patología de base y comorbilidad asociada.

Conclusión: según los datos analizados, la epidemia de gripe A se comportó como una enfermedad benigna, de sintomatología similar a la gripe estacional. Tan solo el hecho de encontrar clínica gripal en una semana epidemiológica de alta incidencia (semanas 43-48) resultaron moderadamente predictores de infección por gripe A.

Palabras clave: Virus Influenza A nH1N1. Pacientes ambulatorios. Atención Primaria. Signos y sintomas. Gripe humana: complicaciones. Epidemiología. Vigilancia epidemiológica.

José Galbe Sánchez-Ventura, galbester@gmail.com

Los autores declaran no presentar conflictos de intereses en relación con la preparación y publicación de este artículo. 
Study of pandemic A nH1N1 influenza (2009-2010). Guiding signs in primary care and comparison with hospitalized cases

Abstract

Objectives: to analyze the prediction's capacity of clinical symptoms to diagnose nH1N1 Influenza in outpatients who were chosen by Aragon's Sentinel surveillance Network. To compare outpatients with hospitalized cases during influenza A virus pandemic (2009-2010).

Methods: retrospective study of a randomized group of patients with symptoms of influenza who had laboratory-confirmation by PCR-RT and of all patients admitted to the reference hospital of Aragon. Comparison of epidemiological and clinical characteristics in outpatients and between outpatients and hospital cases.

Results: there were no clinical differences between the laboratory-confirmed by PCR-RT cases and the other outpatients, so it was not possible to find defining symptoms of infection. Hospitalized patients were younger, with higher percentage of underlying disease and comorbidity.

Conclusions: the epidemic of influenza A behaved as a benign disease, symptoms were similar to seasonal influenza. The clinical finding of having influenza symptoms in a high incidence week (weeks 43-48) was moderately predictive of influenza A infection.

Key words: Influenza A Virus, nH1N1 Subtype. Outpatients. Primary Care. Signs and symptoms. Complications. Epidemiology. Sentinel surveillance.

\section{Introducción}

En marzo de 2009 emergió una epidemia en México causada por un nuevo virus, el virus Influenza A nH1N1, cuya primera transmisión se cree que tuvo lugar al menos varios meses antes del primer brote. Rápidamente se propagó a nivel mundial. En vistas del gran número de personas y países afectados, la Organización Mundial de la Salud declaró el nivel 6 de pandemia el 11 de junio de 20091,2. Un rasgo característico fue la propensión del virus a afectar a niños, adultos jóvenes y embarazadas, especialmente con enfermedad cardiaca o respiratoria de base. Todo ello favoreció que las industrias farmacéuticas se esforzaran en el desarrollo de vacunas para este virus ${ }^{3}$.
El número real de casos de gripe en la epidemia del año 2009-2010 a nivel mundial se desconoce, ya que en la mayoría de los casos se diagnosticaron clínicamente sin prueba de laboratorio confirmatoria; es probable que el número total sea superior a los diez millones de afectados ${ }^{3}$.

La dificultad de realizar el diagnóstico de gripe únicamente con criterios clínicos era un factor limitante para un posible tratamiento con fármacos antivirales. Donde más se ponía de manifiesto este hecho era en los centros de Atención Primaria, donde el uso rutinario de test de diagnóstico rápido ante todo paciente febril era inviable. Hasta el año 2009, la mayor parte del conocimiento sobre la 
sintomatología gripal provenía de estudios sobre pacientes hospitalizados. Esta circunstancia podía distorsionar la sintomatología real de la gripe en la población infantil ${ }^{4}$. Existen estudios que revelan que, en pacientes hospitalizados, tanto los síntomas gastrointestinales, que presentaban un tercio de los pacientes, como la dificultad respiratoria son mucho más frecuentes que en los ambulatorios, aunque en ambos grupos se relacionan con otitis media aguda hasta en un $40 \%$. Los síntomas parecían ser más importantes en los pacientes de menor edad. Se observa que, en años anteriores, los niños hospitalizados por gripe estacional presentaban patología de base comparable con adultos de riesgo y pacientes mayores de 65 años 5 .

La sintomatología diagnóstica de gripe en adultos (fiebre, mialgia y cefalea) difiere en los niños, ya que en estos la cefalea y la mialgia no son síntomas típicos por la incapacidad de referirlo en la anamnesis. La tos no aparece en todos los casos $^{4}$. Algunos estudios proponen la tríada de tos, cefalea y faringitis como predictora de gripe ${ }^{5}$.

Todavía son más escasos los estudios realizados en niños. En un estudio realizado con 2470 adolescentes y adultos con gripe, los signos y síntomas que predecían mejor el diagnóstico de gripe confirmada por métodos microbiológicos fueron la tos y la fiebre, con una sensibilidad del $64 \%$ y una especificidad del $67 \%^{6}$. En niños, las cefaleas y las mialgias solo estuvieron presentes en una minoría de pacientes según otros estu$\operatorname{dios}^{6}$.

Todos los países pusieron en marcha planes de contingencia para poder afrontar situaciones de elevado impacto poblacional. Dentro de nuestro país, cada comunidad autónoma, según su respectivo Servicio de Salud Pública, escogió la definición de "caso sospechoso" de gripe. De manera global, se recogieron tres tipos de casos: el primero, definido por un conjunto de manifestaciones clínicas; el segundo, los casos de infección grave o neumonía, y el tercero, los casos de fallecimiento por una enfermedad respiratoria aguda de causa no aclarada. Estudiando las diferentes definiciones de caso sospechoso usadas en el territorio español, se observan diferencias en la inclusión del periodo de incubación, la consideración del inicio brusco como hallazgo, el punto de corte de temperatura axilar $\left(38^{\circ} \mathrm{Co} 37,5^{\circ} \mathrm{C}\right)$ así como el número de "síntomas respiratorios" que debía valorarse, los síntomas incluidos en el ítem "otras manifestaciones clínicas", la forma de descartar "otras causas" de la sintomatología del 
paciente, el requisito de la existencia de viaje a zona con epidemia, contacto estrecho con caso probable o confirmado, trabajar en un laboratorio o ser manipulador de material potencialmente contaminado. Estas diferencias en la definición han podido afectar la homogeneidad de los casos registrados $y$, secundariamente, a la validez final de los mismos ${ }^{7}$. En el caso de la comunidad autónoma de Aragón, se escogió la elegida por The Official Journal of European Union (1 de mayo de 2009), que definía el caso sospechoso de gripe $\mathrm{A} n \mathrm{n} 1 \mathrm{~N} 1$ como aquel paciente que presentaba a) aparición súbita de los síntomas; b) al menos uno de los cuatro síntomas generales siguientes: fiebre o febrícula, malestar general, cefalea o mialgia; c) al menos uno de los tres síntomas respiratorios siguientes: tos, dolor de garganta o disnea, y d) ausencia de otra sospecha clínica.

Desde octubre de 2009 hasta febrero de 2010, la Red Centinela de Aragón (RCA) desarrolló un control epidemiológico a nivel ambulatorio: de todos los pacientes atendidos con clínica sugestiva de gripe (tras la recogida escrita de datos clínicos), se recogían frotis de forma aleatoria que eran enviados al mismo hospital de referencia (Servicio de Microbiología del Hospital Universitario Miguel Servet de Zaragoza).
Los objetivos de este estudio son, por un lado, analizar la validez y la capacidad de predicción de la sintomatología clínica respecto al diagnóstico de gripe $A$ nH1N1 confirmada mediante reacción en cadena de la polimerasa (PCR), y por otro lado, comparar la casuística ambulatoria con los pacientes que requirieron ingreso durante la primera epidemia de gripe A nH1N1 (2009-2010).

\section{Pacientes y métodos}

Se ha realizado un estudio descriptivo retrospectivo mediante:

- Análisis del documento de recogida sistemática de datos que realizó la RCA, cuya población diana eran los niños de 0-14 años que cumplían los criterios de gripe $\mathrm{A} \mathrm{nH} 1 \mathrm{~N} 1$ incluidos en el registro de la RCA y en los que se hubiera realizado frotis $y$ PCR de forma aleatoria (tal y como estaba previsto por la RCA). Temporalidad: registros desde el 1 de octubre de 2009 hasta el 31 de marzo de 2010.

- Estudio de los niños menores de 15 años ingresados por fiebre y/o clínica respiratoria en el Servicio de Infecciosos del Hospital Infantil Miguel Servet de Zaragoza y que tuvieron una prueba de $\mathrm{PCR}$ positiva para gripe A nH1N1. Temporali- 
dad: desde el 1 de agosto hasta el 31 de diciembre de 2009.

En ambos casos, las muestras fueron procesadas y analizadas por el Servicio de Microbiología del Hospital Miguel Servet (Zaragoza, España).

Los datos recogidos en la encuesta de la RCA fueron: edad, sexo, provincia donde se realizó la consulta médica, síntomas y signos clínicos, patología de base, estado vacunal y complicaciones secundarias a la infección. En los datos hospitalarios se añade si recibieron tratamiento con oseltamivir y las pruebas complementarias realizadas, como radiografía de tórax y analítica sanguínea.

Para el análisis estadístico se ha utilizado el paquete estadístico SPSS ${ }^{\circledast}$ versión
15 para Windows ${ }^{\circledR}$ y GSTAT2 $^{\circledR}$. Para comparar proporciones, se ha utilizado la prueba de Chi cuadrado, y para la comparación de medias, la prueba de la T de Student (previa prueba de normalidad con el test de Saphiro-Wilk). El nivel de significación se ha establecido con un error de 0,05.

\section{Resultados}

\section{Casos recogidos de la Red Centinela de Aragón}

Estudio de frotis faríngeo a 129 pacientes, 89 de los cuales $(68,9 \%)$ tuvieron resultado positivo para gripe $\mathrm{A} n \mathrm{n} 1 \mathrm{~N} 1$.

El $80,6 \%$ de los resultados positivos se recogieron entre la semana 43 y la 48 ,

Figura 1. Distribución temporal de los casos de gripe A (nH1N1) de los pacientes recogidos en la Red Centinela de Aragón.

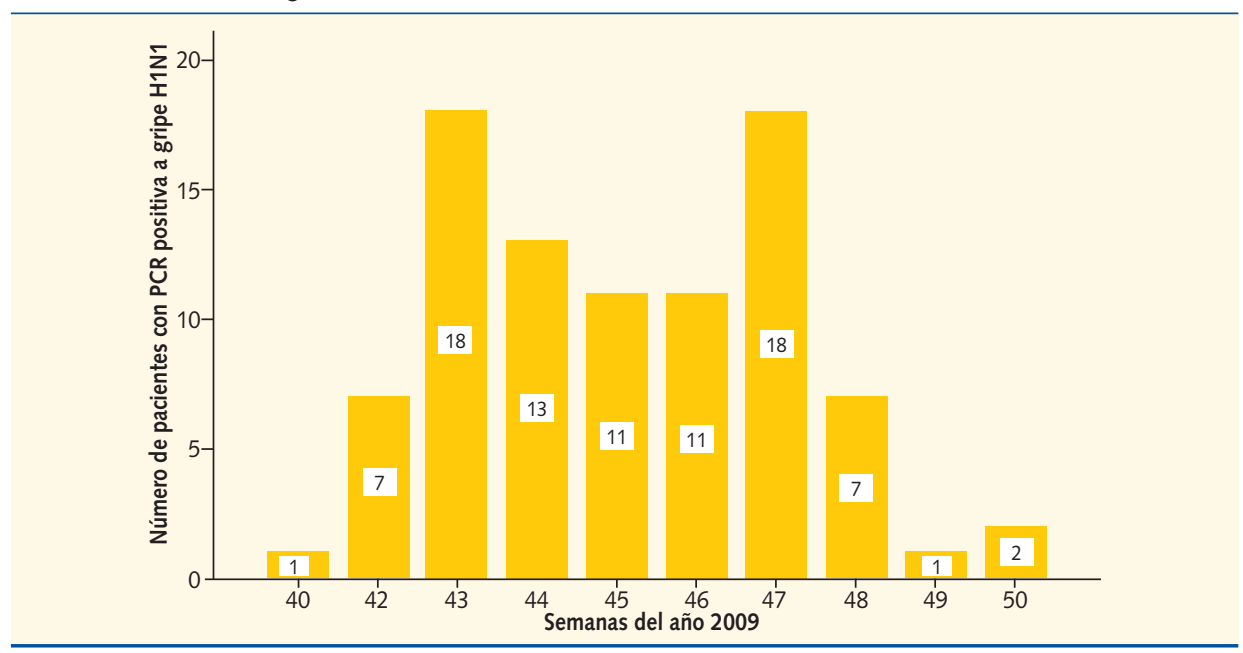




$\begin{aligned} & \text { Tabla 1. Edad de los pacientes con gripe A nH1N1 recogidos de la Red Centinela de Aragón } \\
& \text { hospitalizados }\end{aligned}$
\begin{tabular}{lll}
\hline Edad (años) & $\begin{array}{c}\text { Casos notificados por la RCA } \\
\mathbf{N}=89(\%)\end{array}$ & $\begin{array}{c}\text { Pacientes hospitalizados en el HUMS } \\
\mathbf{N}=49(\%)\end{array}$ \\
\hline Menor de 1 & $1(1 \%)^{*}$ & $18(36,7 \%)$ \\
\hline $1-4$ & $15(16,9 \%)^{*}$ & $11(22,4 \%)$ \\
\hline $5-9$ & $35(39,3 \%)^{*}$ & $8(16,3 \%)$ \\
\hline Mayor de 9 & $38(42,7 \%)^{*}$ & $12(24,5 \%)$ \\
\hline
\end{tabular}

HUMS: Hospital Universitario Miguel Servet, Zaragoza; RCA: Red Centinela de Aragón.

* T de Student: $p<0,001$.

siendo las semanas 43 y 47 en las que más casos positivos se recibieron: 18 $(20,2 \%)$. Detrás de ese rango, la semana 42 le sigue en frecuencia: 7,9\% del total de casos (figura 1). El $32,6 \%$ de los casos procedía de centros de salud de la provincia de Huesca, el $25,8 \%$ de Teruel y el $41,6 \%$ de Zaragoza. En cuanto al sexo de los pacientes, el 53,9\% eran varones, y $46,1 \%$, mujeres. La edad media fue de 8,4 años (desviación estándar [DE]: 3,6 años); los rangos de edad se muestran en la tabla 1.

La técnica de identificación fue PCR en tiempo real (PCR-RT) viral en el $100 \%$ de los casos.

Con respecto a la patología previa, era inexistente en 67 casos (75,3\%); respiratoria en ocho $(9 \%)$, y hubo dos casos con otras patologías previas (quiste hepático y retraso de crecimiento). En la tabla 2 puede verse la sintomatología clínica de los niños que cumplían los criterios epidemiológicos de gripe $A$.

Eran contacto de enfermos de gripe el $48,3 \%$ de los pacientes, en el $21,3 \%$ no constaba y en $30,3 \%$ de los pacientes no existía dicho contacto. De los casos en los que el resultado fue negativo, el $50 \%$ no refería contacto, el 22,5\% sí lo tenían y en el $27,5 \%$ de los casos no constaba.

El estado vacunal era el siguiente: vacunación de gripe estacional en el 3,4\% de los positivos y en el 7,5\% de los negativos. Vacunación frente a gripe $A$ $\mathrm{nH} 1 \mathrm{~N} 1$ en el $1,1 \%$ de los positivos y el 2,5\% de los negativos.

Se realizó una regresión logística introduciendo todos los síntomas que figuran en la hoja de recogida de datos de la RCA; tan solo el hecho de producirse los síntomas en las semanas de mayor incidencia de gripe (43-47) resultó predictivo de tener un frotis positivo. 


\begin{tabular}{lcc}
\hline $\begin{array}{l}\text { Tabla 2. Porcentaje de pacientes ambulatorios notificados a la Red Centinela de Aragón que } \\
\text { presentaron las manifestaciones clínicas sugestivas de gripe. }\end{array}$ \\
$\begin{array}{lcc}\text { Casos notificados (\%) a la RCA } \\
(\mathbf{N}=129)\end{array}$ & $\begin{array}{c}\text { Casos }(\%) \\
\text { con test positivo PCR-RT } \\
(\mathbf{N}=89)\end{array}$ \\
\hline Inicio súbito & 89,2 & 89,9 \\
\hline Fiebre & 87,6 & 87,6 \\
\hline Febrícula & 10 & 8,9 \\
\hline Astenia & 82,2 & 83,1 \\
\hline Cefalea & 64,3 & 64 \\
\hline Artromialgias & 48 & 49,5 \\
\hline Tos & 88,4 & 92,1 \\
\hline Odinofagia & 61,3 & 62,9 \\
\hline Disnea & 10 & 7,9 \\
\hline
\end{tabular}

PCR-RT: reacción en cadena de la polimerasa en tiempo real; RCA: Red Centinela de Aragón.

\section{Casos hospitalarios}

Se han estudiado 49 pacientes; media de edad 5,2 años (DE: 4,6 años; rango dos meses a 15 años) (tabla 1). El 83,7\% de los casos ingresó entre la semana 43 y la 48 , siendo en la semana 46 cuando se ingresó el mayor porcentaje $(22,4 \%)$ (figura 2).

Técnica de identificación: PCR viral en el $100 \%$ de los casos. La sintomatología clínica de los pacientes hospitalizados se muestra en la tabla 3.

En cuanto a las complicaciones, el $49 \%$ de los pacientes presentó neumonía, dos de ellas con empiema y antígeno de neumococo positivo. Como pruebas complementarias, se realizó radiografía de tórax en el $47 \%$ de los casos, se diagnosticaron 22 neumonías, cinco de ellas neumonías multifocales y dos con empiema. No hubo mortalidad en la serie hospitalaria.

\section{Discusión}

Estudiando los casos recogidos por la RCA, se observa que hay un discreto predominio de varones. En cuanto a la edad, la gripe se produce con mayor frecuencia en los niños mayores de cuatro años. La sintomatología clínica referida por los pacientes con gripe A confirmada por PCR-RT no difiere de los que tuvieron un frotis negativo. No se ha encontrado una constelación de síntomas que 
Figura 2. Distribución temporal de los casos de gripe A (nH1N1) de los pacientes hospitalizados. Hospital Miguel Servet de Zaragoza.

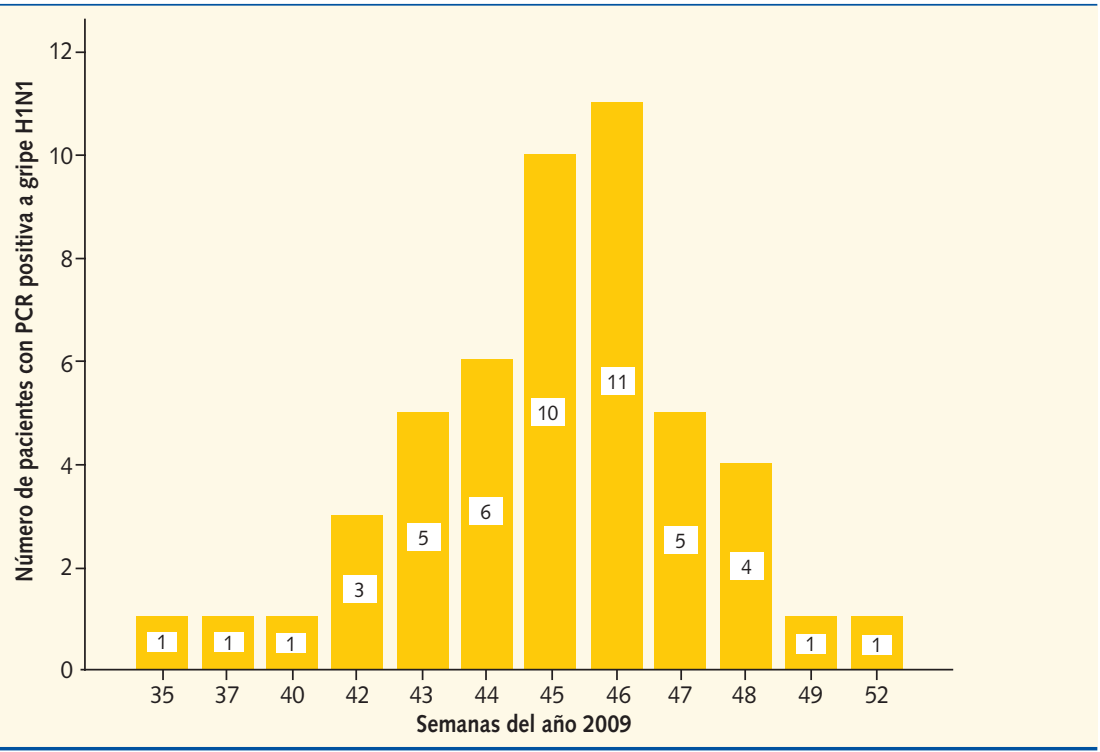

haya resultado predictiva de gripe; tan solo el hecho de producirse el cuadro en una semana de alta incidencia epidemiológica (semanas 43 a 48) junto con los síntomas que definían el caso de gripe podía predecir frotis positivo con PCRRT. Hay que tener en cuenta, no obstante, que la PCR-RT es una prueba adecuada para realizarse en el contexto de una pandemia grave, por su rapidez, así como por su sensibilidad y especificidad adecuadas; sin embargo, está descrito que un porcentaje apreciable de pacientes diagnosticados por PCR en el lavado broncoalveolar tenía una PCR negativa en el frotis faríngeo ${ }^{8}$.
No se encontraron complicaciones graves y solo en un caso se requirió el ingreso hospitalario por presentar meningismo. Existía una baja tasa de vacunación, tanto de la gripe estacional como de la gripe A. Es conocida la baja tasa de vacunación antigripal incluso entre la población de alto riesgo?.

Realizando un estudio comparativo de los casos hospitalarios respecto de los declarados a la RCA, encontramos una diferencia de edad estadísticamente significativa, observándose una mayor tasa de ingresos en los pacientes de menor edad, igual a la descrita por otros autores ${ }^{10}$ (tabla 3). No existen diferencias sig- 


\begin{tabular}{|c|c|c|}
\hline Clínica & $\begin{array}{c}\text { Casos notificados por la RCA } \\
\qquad \mathrm{N}=89(\%)\end{array}$ & $\begin{array}{l}\text { Pacientes hospitalizados en el HUMS } \\
\qquad \mathrm{N}=49(\%)\end{array}$ \\
\hline Fiebre* & 87,6 & 100 \\
\hline Febrícula & 9 & 0 \\
\hline $\begin{array}{l}\text { Astenia o afectación } \\
\text { del estado general* }\end{array}$ & 83,1 & 36,7 \\
\hline Cefalea* & 64 & 16,3 \\
\hline Artromialgias* & 49,4 & 14,3 \\
\hline Tos* & 92,1 & 81,6 \\
\hline Odinofagia* & 62,9 & 2 \\
\hline Disnea* & 7,9 & 20,4 \\
\hline Complicaciones* & 2,2 & 49 \\
\hline \multicolumn{3}{|l|}{ Patología de base* } \\
\hline - Cardiaca & 0 & 8,2 \\
\hline - Respiratoria & 9 & 24,5 \\
\hline - Metabólica & 0 & 2 \\
\hline - Inmunodeficiencia & 0 & 2 \\
\hline
\end{tabular}

HUMS: Hospital Universitario Miguel Servet, Zaragoza; RCA: Red Centinela de Aragón.

${ }^{*}$ Chi cuadrado: $p<0,001$.

nificativas entre ambos sexos. Entre la tología de base en mayor proporción, sintomatología recogida hay menor pro- fundamentalmente respiratoria (asma, porción de cefalea, astenia, mialgia, tos y displasia broncopulmonar) ${ }^{10-12}$. La tasa odinofagia, con diferencias estadística- de complicaciones fue significativamenmente significativas, si bien estos datos te mayor, siendo la neumonía la más frepueden estar infrarrepresentados en la cuente. La evolución fue favorable en anamnesis realizada al ingreso. En los todos los casos, sin existir mortalidad pacientes ingresados se observa una asociada.

mayor proporción de fiebre (100\%) y Si comparamos estos datos de los nidisnea. Estas diferencias también son es- ños hospitalizados con diagnóstico de tadísticamente significativas. Los pa- gripe A con los comunicados por el Hoscientes hospitalizados presentaban pa- pital La Paz de Madrid para el mismo pe- 
riodo de tiempo ${ }^{12}, 128$ casos, las diferencias más importantes están en el predominio de varones ingresados por gripe en este último hospital, así como un mayor porcentaje de pacientes ingresados con tos, dificultad respiratoria, cefalea, mialgias, vómitos y diarreas, así como un mayor porcentaje de pacientes afebriles. Otra característica diferencial de los pacientes ingresados en el Hospital La Paz respecto de los del Hospital Miguel Servet es que los primeros tenían una mayor frecuencia de patologías previas como asma, cardiopatías, inmunosupresión, trastornos neurológicos, trasplantes y otros, y por tanto representaban a un grupo de población con trastornos previos más graves ${ }^{12,13}$. En el Hospital La Paz se administró tratamiento con oseltamivir en mayor proporción que en el Hopital Miguel Servet y se realizaron más ingresos en la Unidad de Cuidados Intensivos. Los pacientes ingresados en La Paz eran asimismo de menor edad ${ }^{14}$. Las diferencias encontradas entre la población de la RCA, la de los niños ingresados por gripe $A$ en el Hospital Miguel Servet y la del Hospital La Paz se deben sin duda a que existe una gradación progresiva en la gravedad y en la complejidad de los casos de gripe según procedan de la RCA, del Hospital Miguel Servet o del Hospital La Paz. La población del estudio de la RCA es una población tomada de la Atención Primaria de Aragón y de la red centinela de esta comunidad, siendo por tanto mucho más representativa de la población real de cara a valorar el verdadero impacto de la gripe A pandémica nH1N1 en nuestra comunidad en el periodo de tiempo analizado.

Respecto a la utilidad de la sintomatología clínica para el diagnóstico de gripe, hay que destacar que la mayoría de los estudios indican una modesta capacidad de predicción de los síntomas tanto de forma aislada como en combinación ${ }^{5,6,15-17}$. También es necesario señalar la similitud de la sintomatología clínica de la gripe estacional con la de la gripe $\mathrm{A}$ pandémica $\mathrm{nH} 1 \mathrm{~N} 1^{18}$.

\section{Conclusiones}

- La epidemia de gripe A se comportó durante 2009 y 2010 en Aragón como una enfermedad autolimitada y benigna, de sintomatología similar a la gripe estacional.

- Hubo una máxima incidencia a los 1-9 años. En el caso de los niños hospitalizados, la incidencia máxima se produjo a los 6-14 años, siendo los niños menores de cinco años los que presentaron mayor tasa de ingreso.

- El pico epidemiológico de esta gripe se produjo aproximadamente diez 
semanas antes que el pico de gripe estacional en años previos, por lo que desplazó las cepas de virus de gripe estacional, en lugar de producirse una suma de casos.

- No existió una combinación de síntomas que resultase útil en la predicción de infección por gripe $A$ $\mathrm{nH} 1 \mathrm{~N} 1$ en periodos de baja incidencia de la enfermedad. Tan solo el hecho de producirse el cuadro en una semana epidemiológica de alta incidencia (semanas 43-48) junto con la presencia de síntomas definitorios de caso clínico resultó moderadamente predictor de tener un frotis positivo.

- Los síntomas fueron muy similares entre los casos notificados a la RCA, sin que se diferenciaran los casos con frotis positivo o negativo.
- No existieron complicaciones graves entre los niños notificados a la RCA en el periodo de tiempo analizado (de 09/10/2009 al 12/02/2010). Tan solo fue necesaria la hospitalización en un caso por meningismo.

- Los casos hospitalarios presentaron mayor tasa de complicaciones, fundamentalmente neumonía, aunque todos los casos evolucionaron favorablemente.

- La pandemia por gripe A nH1N1 resultó menos virulenta de lo inicialmente esperado.

\section{Agradecimientos}

Al Departamento de Vigilancia Epidemiológica del Gobierno de Aragón y a la Red Centinela perteneciente al mismo organismo, sin cuya colaboración no hubiese sido posible este trabajo.

\section{Bibliografía}

1. Sullivan SJ, Jacobson M, Dowdlw WR, Poland GA. 2009 nH1N1 Influenza. A review. Mayo Clin Proc. 2010;85(1):64-76.

2. Shlomai A, Nutman A, Kotlovsky T, Schechner $V$, Carmeli $Y$, Guzner-Gur H. Predictors of pandemic (nH1N1) 2009 virus positivity and adverse outcomes among hospitalized patients with a compatible syndrome. Isr Med Assoc J. 2010;12(10):622-7.
3. Girard MP, Tam JS, Assossou OM, Kieny MP. The 2009 A (nH1N1) influenza virus pandemic: A review. Vaccine. 2010;28(31):4895-902.

4. Silvennoinen $H$, Peltola $V$, Lehtinen $P$, Vainionpää $R$, Heikkinen $T$. Clinical presentation of influenza in unselected children treated as outpatients. Pediatr Infect Dis J. 2009;28(5):372-5.

5. Friedman MJ, Attia MW. Clinical predictors of influenza in children. Arch Pediatr Adolesc Med. 2004;158(4):391-4. 
6. Grupo de Trabajo de Pediatría basada en la evidencia de la AEP (GT-PBE). Informe Técnico en Pediatría sobre Gripe Pandémica $\mathrm{nH} 1 \mathrm{~N} 1$ [en línea] [actualizado el 11/01/2010; consultado el 25/04/2011]. Disponible en www.aeped.es/documentos/informe-tecnico-en-pediatria-so bre-gripe-pandemica-h1n1

7. Hernández Merino A. Nueva gripe [A(nH1N1) 2009]: definición de caso sospechoso. Revisión de la concordancia en los criterios de definición de caso utilizados en las distintas comunidades autónomas españolas. Rev Pediatr Aten Primaria. 2009;11:383-98.

8. Blyth CC. Rapid test Sensitvity for novel Swine-Origin Influenza $A$ (nH1N1) in Humans. $N$ Engl J Med. 2009;36:2493.

9. Domínguez Cajal MM, Arriba Muñoz A, Escosa García L, García Iñiguez JP, Biosca Pámies M, García Sánchez N. Cobertura vacunal antigripal en niños de riesgo durante 2007-2008 en un Centro de Atención Primaria en España. Rev Pediatr Aten Primaria. 2009;11:399-411.

10. Jain S, Kamimoto L, Bramley AM, Schmitz AM, Benoit SR, Louie J et al. Hospitalized patients with 2009 nH1N1 influenza in the United States, April-June 2009. N Engl J Med. 2009;361(20): 1935-44.

11. O'Riordan S, Barton M, Yau Y, Read SE, Allen $U$, Tran D. Risk factors and outcomes among children admitted to hospital with pandemic
nH1N1 influenza. CMAJ. 2010;182(1):39-44.

12. Escosa García L, Aracil Santos FJ, De José Gómez MI. Ingresos por infección emergente por virus Influenza 2009 A/nH1N1 en un hospital terciario. Madrid: Universidad Autónoma de Madrid (Diploma de Estudios Avanzados); 2010.

13. Hackett S, Hill L, Patel J, Ratnaraja N, Ifeyinwa $A$, Faroogi $M$ et al. Clinical characteristics of paediatric $\mathrm{nH} 1 \mathrm{~N} 1$ admissions in Birmingham, UK. Lancet. 2009;374(9690):605.

14. Poehling KA, Edwards $K M$, Weinberg $A$, Szilagyi PS, Staat MA, Iwane MK et al. A systematic review of the history and physical examination to diagnose influenza. J Am Board Fam Pract. 2004;17(1):1-5.

15. Ebell MH, Afonso A. A Systematic Review of Clinical Decision Rules For The Diagnosis of Influenza. Ann Fam Med. 2011;9:69-77.

16. Poehling KA, Edwards KM, Weinberg GA, Szilagyi P, Staat MA, Iwane MK et al., for the New Vaccine Surveillance Network. The underrecognized burden of Influenza in young children. N Engl J Med. 2006;355(1):31-40.

17. Call $S A$, Vollenweider MA, Hornung $C A$, Simel DL, McKinney WP. Does this patient have influenza? JAMA. 2005;293(8):987-97.

18. Silvennoinen $H$, Peltola $V$, Lehtinen $P$, Vainionpää $R$, Heikkinen T. Clinical Presentation of Influenza in unselected Children Treated as Outpatients. Pediatr Infect Dis J. 2009;28:372-5. 